\title{
PAPR Reduction of OFDM System with Biorthoganoal Wavelet Transforms
}

\author{
Palle Jagadeeswara Rao ${ }^{1}$ | Dr. A S Srinivasa $\mathrm{Rao}^{2}$ \\ ${ }^{1} \mathrm{PG}$ Scholar, Department of Electronics and Communication, Aditya Institute of Technology and Management (A), \\ Tekkali, AP, India \\ ${ }^{2}$ Professor, Department of Electronics and Communication, Aditya Institute of Technology and Management (A), Tekkali, \\ AP, India
}

To Cite this Article

Palle Jagadeeswara Rao and Dr. A S Srinivasa Rao, "PAPR Reduction of OFDM System with Biorthoganoal Wavelet Transforms", International Journal for Modern Trends in Science and Technology, Vol. 07, Issue 02, February 2021, pp.-17-22.

Article Info

Received on 12-January-2021, Revised on 26-January-2021, Accepted on 29-January-2021, Published on 01-February-2021.

\section{ABSTRACT}

Orthogonal Frequency Division Multiplexing (OFDM) and Multiple Input and Multiple Output (MIMO) are two main techniques employed in 4th Generation Long Term Evolution (LTE). In OFDM multiple carriers are used and it provides higher level of spectral efficiency as compared to Frequency Division Multiplexing (FDM). In OFDM because of loss of orthogonality between the subcarriers there is intercarrier interference (ICI) and intersymbol interference (ISI) and to overcome this problem use of cyclic prefixing (CP) is required, which uses 20\% of available bandwidth. Wavelet based OFDM provides good orthogonality and with its use Bit Error Rate (BER) is improved. Wavelet based system does not require cyclic prefix, so spectrum efficiency is increased. It is proposed to use wavelet transform including biorthoganoal wavelet transform with OFDM systems. This approach will reduce PAPR in the OFDM system effectively. We will design this model with different modulation Techniques like QPSK and QAM and compare the BER results.

Keywords-LTE; OFDM; DFT; Wavelet; BER

\section{INTRODUCTION}

A set of requirements are specified for 4 th generation of cellular systems by International Telecommunication Union Radio Communication Sector (ITU-R). The requirement of data rate was specified in International Mobile Telecommunications Advanced project (IMT-Advanced). 3rd Generation Partnership Project (3GPP) was established in 1998 [1]. 3GPP started working on the LTE project to define the Radio Access Network (RAN) and core network [1]. 3GPP's candidate for 4G was LTE-Advanced. OFDM is one of the main techniques employed in LTE to enhance the data rate. Spectrum efficiency and flexible utilization of spectrum is highly required today for different wireless communication related applications. In multicarrier communication the main idea is to divide the data into several streams and using them to modulate different carriers. The two main advantages of multicarrier communication are, first one is there is no requirement of signal enhancement for noise which is required in single carrier because of the equalizers and second is because of long symbol duration reduced effect of fading [2]. In OFDM subcarriers used are orthogonal to each other. Orthogonality causes the subcarriers to overlap in frequency domain, so the bandwidth efficiency is obtained without any ICI [3]. Wavelet transform is used to analyze signals by 
the coefficients of wavelets in both time and frequency domain. ISI is between successive symbols of same sub-carrier and ICI is among different signals at different subcarriers. Both are avoided by use of cyclic prefixing which causes power loss and bandwidth inefficiency in DFT based OFDM [3]. There are some recent works on wavelet based OFDM systems. In [4], author compares performance of wavelet based OFDM system in different channel condition using hear wavelets with DFT based OFDM system. In [5], author have compared performance of DFT based OFDM and wavelet based OFDM by using BER as performance parameter and Rayleigh fading channel. In [6], author presented wavelet packet modulation for OFDM and presented the performance comparison of both the techniques. In [10], author have presented a comparison on different multicarrier modulations and used ISI/ICI for performance measurement. In [11], wavelet modulation has been proposed for multicarrier communication. In [12], author proposed multimode transmission using wavelet modulation and OFDM. In this paper we have compared the performance of wavelets based OFDM system with performance of conventional OFDM system for different LTE modulation techniques. For wavelet based system we have used daubechies2 and haar wavelets. Additive White Gaussian Noise (AWGN) channel is used for transmission.

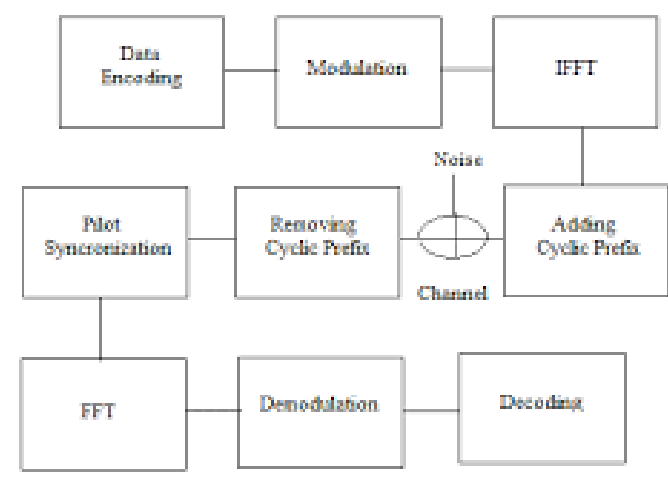

Fig.1 OFDM transceiver system

\section{BASICS OF OFDM}

\section{A. FOURIER-BASED OFDM (FFT-OFDM)}

An OFDM transceiver system is shown in Fig. 1. The inverse and forward transform blocks are concerned in more attentions since they can be FFT-based or DWT-based OFDM. The system model for FFT-based OFDM will not be discussed in detail as it is well known in the literature. Thus, we merely present a brief description about it. The data generator produces $\mathrm{dk}$ in random binary form. It is first being processed by a constellation mapping. M-ary QAM modulator is used for this work to map the raw binary data to appropriate QAM symbols Xm. These symbols are then input into the IFFT block. This involves taking $\mathrm{N}$ parallel streams of QAM symbols ( $\mathrm{N}$ being the number of sub-carriers used in the transmission of the data) and performing an IFFT operation on this parallel stream.

\section{B. CONVENTIONAL OFDM SYSTEM:}

For conventional OFDM system sinusoids of DFT form an orthogonal basis function set. In DFT the transform correlates its input signal with each of sinusoidal basis function [13], here orthogonal basis functions are the subcarriers used in OFDM. At the receiver the signals are combined to obtain the information transmitted. Practically, Fast Fourier Transform (FFT) and Inverse Fast Fourier Transform (IFFT) are used for the implementation of the OFDM system because less number of computations required in FFT and IFFT. Multiple replicas of the signal are received at the receiver end because of the time dispersive nature of the channel, so frequency selective fading results and to reduce this interference guard interval is used, which is called cyclic prefix [14]. Cyclic prefix is copy of some fraction of symbol end. As long as the channel delay spread remains within the limit of the cyclic prefix there would not be any loss in orthogonality. For LTE, in the downlink data of different users is multiplexed in frequency domain and access technique is called Orthogonal Frequency Division Multiple Access (OFDMA) [15]. In the uplink of the LTE access technique used is Single Carrier-Frequency Division Multiple Access (SC-FDMA). High Peak Average Power Ratio (PAPR) occurs due to random constructive addition of subcarriers and results in spectrum spreading of signal leading to adjacent channel interference. So power linearization techniques and compression point amplifier need to be used to overcome this problem. Due to single carrier modulation effect of ISI will be high in uplink and to overcome from its effect low complexity equalizer will be required but SC-FDMA is not sensitive to frequency offset and Doppler shift [14]. 


\section{WAVELET BASED OFDM SYSTEM}

In previous works use of Discrete Fourier Transform was proposed for the implementation of OFDM [2]. Wavelet transform show the potential to replace the DFT in OFDM. Wavelet transform is a tool for analysis of the signal in time and frequency domain jointly. It is a multi-resolution analysis mechanism where input signal is decomposed into different frequency components for the analysis with particular resolution matching to scale [8].

Using any particular type of wavelet filter the system can be designed according to the need and also the multi resolution signal can be generated by the use of wavelets. By the use of varying wavelet filter, one can design waveforms with selectable time/frequency partitioning for multi user application [6]. Wavelets possess better orthogonality and have localization both in time and frequency domain [16]. Because of good orthogonality wavelets are capable of reducing the power of the ISI and ICI, which results from loss of orthogonality. To reduce ISI and ICI in conventional OFDM system use of cyclic prefix is there, which uses $20 \%$ of available bandwidth, so results in bandwidth inefficiency but this cyclic prefix is not required in wavelet based OFDM system. Complexity can also be reduced by using wavelet transform as compared with the Fourier transform because in wavelet complexity is $\mathrm{O}[\mathrm{N}]$ as compared with complexity of Fourier transform of $\mathrm{O}$ [N $\log 2 \mathrm{~N}]$ [17]. Wavelet based OFDM is simple and the DFT based OFDM is complex. Wavelet based OFDM is flexible as well and because better orthogonality is provided by it, there is no any need of cyclic prefixing in wavelet based OFDM, which is required in DFT based OFDM to maintain orthogonality so wavelet based system is more bandwidth efficient as compared with the DFT based OFDM.

In discrete wavelet transform (DWT), input signal presented will pass through several different filters and will be decomposed into low pass and high pass bands through the filters. During decomposition the high pass filter will remove the frequencies below half of the highest frequency and low pass filter will remove frequencies that are above half of the highest frequency. The decomposition halves the time resolution because half of the samples are used to characterize the signal similarly frequency resolution will be doubled and this decomposition process will be repeated again for obtaining the wavelet coefficients of required level. Two types of coefficients are obtained through processing, first ones are called detailed coefficients obtained through high pass filter and second ones are called coarse approximations obtained through low pass filter related with scaling process. After passing the data through filters the decimation process will be performed. The whole procedure will continue until the required level is obtained.

This decomposition can be given as

$$
\begin{aligned}
\mathrm{Y}_{\text {high }}[\mathrm{k}]= & \sum_{\mathrm{n}} \mathrm{x}[\mathrm{n}] \mathrm{g}[2 \mathrm{k}-\mathrm{n}] \\
& \mathrm{y}_{\text {low }}[\mathrm{k}]=\sum_{\mathrm{n}} \mathrm{x}[\mathrm{n}] \mathrm{h}[2 \mathrm{k}-\mathrm{n}]
\end{aligned}
$$

Where $\mathrm{x}[\mathrm{n}]$ is the original signal, $\mathrm{g}[\mathrm{n}]$ is impulse response of half-band high pass filter and $h[n]$ is impulse response of half-band low pass filter. $\mathrm{y}_{\text {high }}[\mathrm{k}]$ and $\mathrm{y}_{\text {low }}[\mathrm{k}]$ are obtained after filtering and decimation by a factor of 2 . In inverse discrete wavelet transform (IDWT), the reverse process of decomposition is performed, so here firstly upsampling is done then the signal is passed through the filters. The data obtained after filtering is combined to obtain reconstructed data. Number of levels during reconstruction will be same as that of the decomposition.

\section{SYSTEM MODEL}

\section{A. PROPOSED WAVELET BASED OFDM DESIGN}

As shown in figure 2, in this proposed model we are using IDWT and DWT at the place of IDFT and DFT. AWGN channel is used for transmission and cyclic prefixing is not used. Here first of all conventional encoding is done followed by interleaving then data is converted to decimal form and modulation is done next. After modulation the pilot insertion and sub carrier mapping is done then comes the IDWT of the data, which provides the orthogonality to the subcarriers. IDWT will convert time domain signal to the frequency domain. After passing through the channel on the signal DWT will be performed and then pilot synchronization where the inserted pilots at the transmitter are removed then the demodulation is done. Demodulated data is converted to binary form and the de-interleaved and decoded to obtain the original data transmitted. 


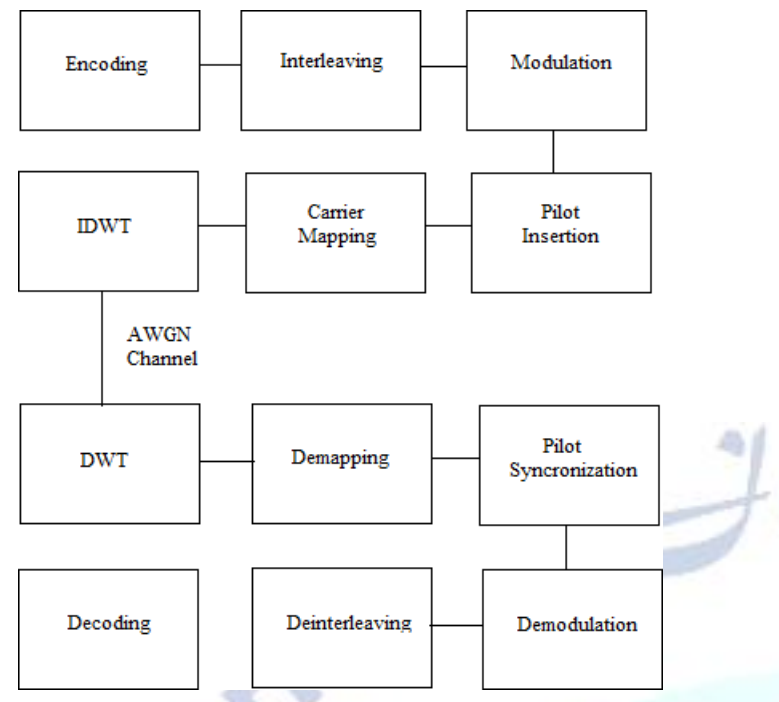

Fig. 2. Wavelet based proposed OFDM system design

\section{B. BER PERFORMANCE EVALUATION}

By using MATLAB performance characteristic of DFT based OFDM and wavelet based OFDM are obtained for different modulations that are used for the LTE, as shown in figures 3-5. Modulations that could be used for LTE are QPSK, 16 QAM and 64 QAM (Uplink and downlink). QPSK does not carry data at very high speed. When signal to noise ratio is of good quality then only higher modulation techniques can be used. Lower forms of modulation (QPSK) does not require high signal to noise ratio.

For the purpose of simulation, signal to noise ratio (SNR) of different values are introduced through AWGN channel. Data of 9600 bits is sent in the form of 100 symbols, so one symbol is of 96 bits. Averaging for a particular value of SNR for all the symbols is done and BER is obtained and same process is repeated for all the values of SNR and final BERs are obtained. Firstly, the performance of DFT based OFDM and wavelet based OFDM are obtained for different modulation techniques. Different wavelet types daubechies2 and haar is used in wavelet based OFDM for QPSK, 16-QAM, 64-QAM.

\section{RESULTS AND DISCUSSION}

A closed form formula is derived for the bit $\begin{array}{ll}\text { error rate } & \text { (BER) } \\ \text { orthogonal-frequency-division-multiplexing }\end{array}$ (OFDM) with M-ary differential-phase-shift-keying (MDPSK) systems in frequency-selective Rayleigh and Rician fading channels with diversity reception. New BER curves are obtained as a function of the rms delay spread of the diffused component for three different types of delay profiles: (1) one-sided exponential, (2) uniform and (3) double spike profiles. Both slow and fast fading conditions are considered. It is shown that the existence of a strong line-of-sight (LOS) component and the use of reception diversity can effectively improve transmission performance Similarity and difference between orthogonal frequency division multiplexing passive optical network(OFDM-PON) and wavelength division multiplexing passive optical network(WDM-PON) are discussed ,indicating that WDM-PON is actually a kind of physical layer technique which can perform the functions of data transmission system and network only by combining itself with some media access control(MAC) technique. In contrast, OFDM-PON is an optical access network working with the technique of orthogonal frequency division multiple accesses. Even its optical network units(ONUs) occupy different optical spectra, it is not a WDM-PON. Concept of OFDM-PON is clarified from two viewpoints: the optical beat interference $(\mathrm{OBI})$ and $\mathrm{MAC}$, to promote the development and standardization of OFDM-PON. There has been increased interest in high data rate indoor communications systems using orthogonal frequency division multiplexing (OFDM). This paper details both simulation and experimental results, regarding the sensitivity of such signals to amplifier nonlinearities.

The OFDM make use of a sophisticated divide-and-conquer methodology to transmit high speed frequency-selective channels.23 It is a multicarrier block modulation scheme wherein the whole available bandwidth is divided into different sequence of symbols of low data rates and then modulated simultaneously by multiple partially overlapped subcarriers which are orthogonal to each other and transmitted in parallel form to achieve high aggregate data rates and bandwidth efficiency. Flat frequency response is generated in individual sub-carriers with constricted bandwidth in sub-channel, avoiding complex equalization in time-domain. Thus, frequency-selective channel is transformed into individual flat-fading channel enhancing receiver signal9 robust to multi-path fading and bit error rate. Orthogonality 5 in the carriers also eliminates the inter-carrier interference. Insertion of spectral guard bands for partially overlapped sub-carriers provides higher spectral efficiency with respect to conventional Frequency Division Multiplexing (FDM). Digital 
data is transformed and mapped in accordance to sub-carrier amplitude and phase. Spectral data in mapped to the time domain using Inverse Discrete Fourier Transform. The implementation of IFFT in place of IDFT further adds to computational efficiency at the cost slight increase in operational infrastructure. Hence IFFT/FFT is now deployed in all practical systems. Reverse operation is performed at the receiver section, where RF signals are added to baseband proceeded by FFT that analyses the the received signal in frequency domain. Digital data is generated out of the subcarrier's amplitude and phase. FFT and IFFT are complementary in nature 24 .

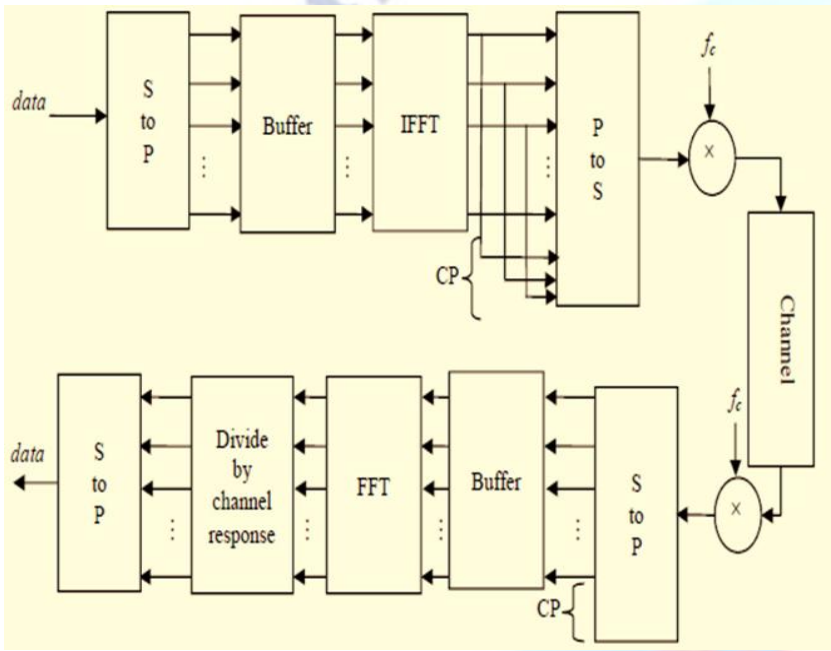

Fig.3: Block Diagram of OFDM24

Simulation results of PAPR reduction using different modulation techniques are given below figures.

A) BER performance of wavelets DFT based OFDM system using 16QAM

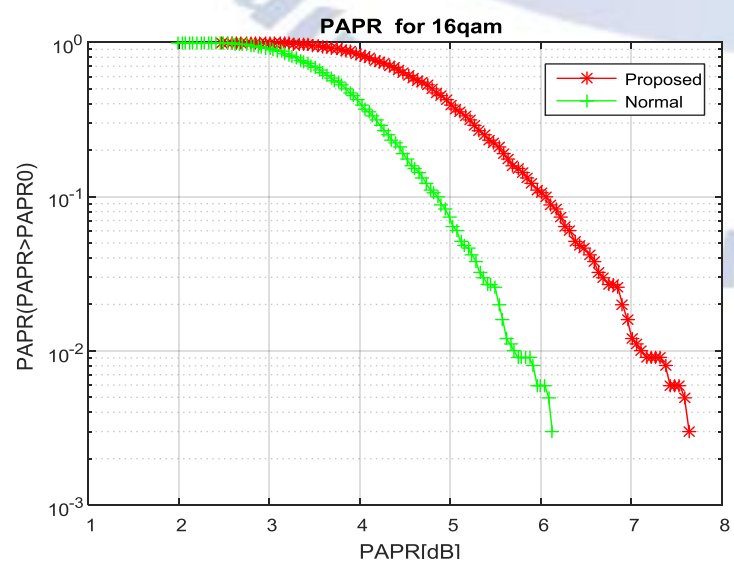

Fig.4. PAPR reduction using 16QAM
B) Show the BER performance of OFDM system with wavelets using 64QAM

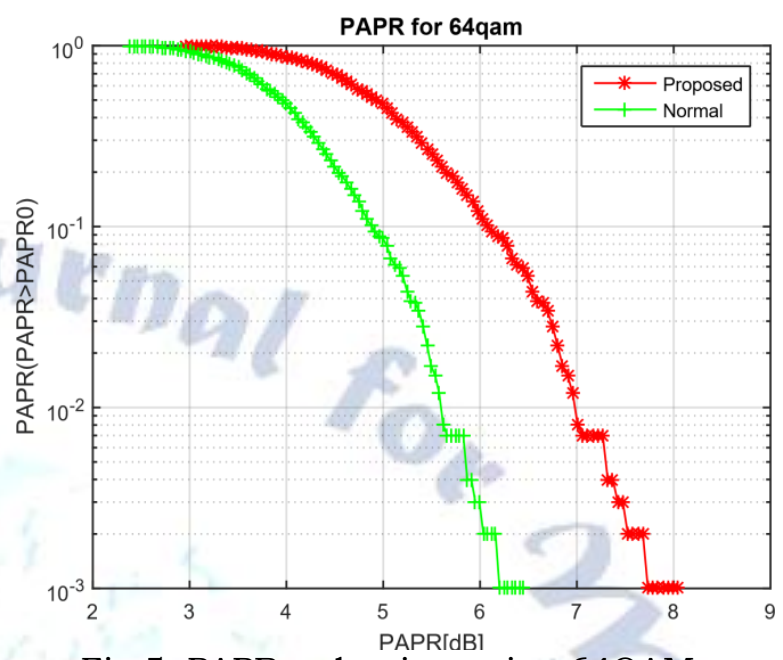

Fig.5. PAPR reduction using 64QAM

\section{v. CONCLUSION}

As a conclusion, it can be stated that PRP-OFDM is anevolution of OFDM suitable in the context of mobility where low-complexity channel estimation with a low pilot overhead is welcome. IOTA-OFDM follows a different approach, since no prefix/postfix sequence is any more required. It therefore aims a an increase in throughput, while channel tracking still requires

pilot tones. Note that most known enhancements of CP-OFDM, such as spreading techniques (MC-SS), are compatible with ZP-,PRP- and IOTA-OFDM.

\section{FUTURE SCOPE}

Exploration as well as up gradation to $5 \mathrm{G}$ mobile wireless technologies have been the current topic of research in both the academia and industries. Though Code-Division Multiple Access (CDMA) was used extensively in the Third Generation (3G) technologies, it had certain disadvantages like Inter-Symbol-Interferences and High power consumptions. The CDMA was replaced with OFDM as it possessed higher ease of implementation, resistant to external interference with faster high data-rate. The OFDM is a multicarrier orthogonal digital communication scheme, it divides the whole available bandwidth into many streams of low data rates that are then modulated simultaneously by multiple carriers. The modulation schemes deployed defines the spectral efficiency and application of the waveform in wireless communication standards. 1 The OFDM enjoys superiority in a number of technological and 
performance aspects. To mention a few, it has lowest complexity when compared to other waveforms due to the use of Fast Fourier Transform (FFT) / Inverse Fast Fourier Transform( IFFT), highest bandwidth efficiency, higher resistance to frequency selective fading, exceptional symbol structure and simplified synchronization. The OFDM does possess two major drawbacks, very high Peak to Average Power Ratio and Out-Of-Band (OOB) emissions that significantly reduce system performance and throughput. Others include Cyclic Prefix and bigger side lobes that limits the spectrum efficiency. Even though OFDM marks a tremendous leap in the technological advancement in $4 \mathrm{G}$, the above mentioned defects overshadows it to be deployed for $5 \mathrm{G}$ standards. Research studies show the proposal and development of other multicarrier waveforms in recent years to improve $\mathrm{PAPR}$ and $\mathrm{OOB}$ emissions for $5 \mathrm{G}$.

\section{REFERENCES}

[1] Anuradha, Naresh Kumar, "BER analysis of conventional and wavelet based OFDM in LTE using different modulation techniques". Proceeding of 2014 RAECS UIET Punjab University Chandigarh, 06-08 March, 2014 IEEE.

[2] G. Sailakshmi, K. Sudha, "BER analysis of wavelet based OFDM using different modulation techniques", International Journal of Science and Research, vol. 4 issue 5, May2015

[3] S. Padmaja, S. Chakri Sreedhar, "BER analysis of conventional and wavelet based OFDM In LTE using different modulation techniques", International Journal of Eminent Engineering Technologies, vol. 2 issue 4, May 2015

[4] Pargtee Tomar, Mitra Sharma, Bhawani Shankar Chaudhary, "Bit Error Rate (BER) Analysis of Conventional OFDM (DFT-OFDM) and Wavelet Based OFDM (DWT-OFDM)", International Journal on Recent and Innovation Trends in Computing and Communication, vol.3, issue 1, pp.423-426, January 2015.

[5] K. Volkan, K. Oguz, "Alamouti coded wavelet based OFDM for multipath fading channels", IEEE Wireless telecommunications symposium, pp.1-5, April 2009.

[6] G. Mahesh Kumar, S. Tiwari, "Performance evaluation of conventional and wavelet based OFDM system", International journal of electronics and communications, Elsevier, vol. 67, no. 4, pp. 348-354, April 2013.

[7] J. Antony, M. Petri, "Wavelet packet modulation for wireless communication", Wireless communication \& mobile computing journal, vol. 5, no. 2, pp. 1-18, March 2005

[8] Mr. Sivanagaraju and Dr. Siddaiah "Comprehensive Analysis of BER and SNR in OFDM Systems" International Journal of Innovative Research in Computer and Communication Engineering, Vol. 2, Issue 2, February 2014

[9] Mr. Sumit Dalal, Mr Pulkit Berwal, Electronics \& Communication Dept., Ganga Technical Campus,
Soldha Bahadurgarh, "A BER Performance Analysis of Shift Keying Technique with MMSE/MLSE estimation in Fading domain" IJIRAE, June 2014

[10] M. Divya "Bit Error Rate Performance of BPSK Modulation and OFDM-BPSK with Rayleigh Multipath Channel” IJEAT, April 2013. 